\title{
Additional L-Carnitine Reduced the Risk of Hospitalization in Patients with Overt Hepatic Encephalopathy on Rifaximin
}

\author{
Kousuke Kubota $^{a}$ Haruki Uojima ${ }^{a, b}$ Xue Shao ${ }^{a, c}$ Shuichiro Iwasaki ${ }^{a}$ \\ Hisashi Hidaka ${ }^{a}$ Naohisa Wada ${ }^{a}$ Takahide Nakazawa $^{a}$ Akitaka Shibuya ${ }^{a}$ \\ Makoto Kako ${ }^{a}$ Wasaburo Koizumi ${ }^{a}$ \\ ${ }^{a}$ Department of Gastroenterology, Internal Medicine, Kitasato University School of Medicine, Sagamihara, \\ Japan; ' Department of Gastroenterology, Shonan Kamakura General Hospital, Kamakura, Japan; 'Department of \\ Hepatopancreatobiliary Medicine, The Second Hospital of Jilin University, Changchun, China
}

\author{
Keywords \\ Hepatic encephalopathy · L-Carnitine - Liver cirrhosis · \\ Portosystemic shunt · Rifaximin
}

\begin{abstract}
Background: Data regarding the additional effect on the recurrence of hepatic encephalopathy (HE) after oral L-carnitine administration are scarce. Objective: This study aimed to assess the additional effects of L-carnitine in patients who were receiving rifaximin for HE. Methods: This randomized study comprised a screening visit and a 12-week treatment period. Patients who fulfilled the eligibility criteria were randomized to either group $A$ (additional rifaximin) or group $B$ (additional L-carnitine and rifaximin). Group A received 1,200 $\mathrm{mg} /$ day of rifaximin. Group B received 1,500 mg/day of Lcarnitine and rifaximin at $1,200 \mathrm{mg} /$ day. The endpoints were the changes in the portal systemic encephalopathy (PSE) index and the admission rate from the baseline for the duration of the study in both groups. Results: Eighty-three patients were randomized to either group $A(n=42)$ or group $\mathrm{B}(n=41)$. In group A, the PSE index decreased from $0.35 \pm$
\end{abstract}

0.09 at baseline to $0.27 \pm 0.11$ on the final evaluation day $(p=0.001)$. In group $\mathrm{B}$, the PSE index decreased from $0.37 \pm$ 0.09 at baseline to $0.24 \pm 0.11$ on the final evaluation day $(p=0.001)$. Although there was not a significant reduction in the PSE index in group A compared to that in group B ( $p=$ 0.202 ), the admission rates were $30.9 \%$ and $9.8 \%$ in groups $A$ and $B$, respectively. Additional L-carnitine significantly reduced the admission rate $(p=0.028)$. Conclusion: $L$-Carnitine addition reduced the risk of hospitalization for patients who received rifaximin for $\mathrm{HE}$.

(c) 2021 The Author(s)

Published by S. Karger AG, Basel

\section{Introduction}

Hepatic encephalopathy (HE) is characterized by reversible neuropsychiatric abnormalities in patients with severe liver dysfunction and/or with a portosystemic shunt (PSS) [1]. HE has a poor influence on the quality of life (QOL) in patients with cirrhosis. Especially, many patients with HE complain of sleep disturbance and excessive daytime sleepiness. Sleep-wake cy- karger@karger.com www.karger.com/ddi

Karger $\stackrel{\text { ' }}{5}$ BOPEN ACCESS
(C) 2021 The Author(s)

Published by S. Karger AG, Basel

This is an Open Access article licensed under the Creative Commons Attribution-NonCommercial-4.0 International License (CC BY-NC) (http://www.karger.com/Services/OpenAccessLicense), applicable to the online version of the article only. Usage and distribution for commercial purposes requires written permission.
Correspondence to:

Haruki Uojima, kiruha@ kitasato-u.ac.jp 
cle changes extremely lower QOL. The impairment of QOL leads to higher mortality and poor daily functioning in patients with cirrhosis who remain challenging to treat [2].

The precise molecular mechanisms of HE are unclear. Neuropsychiatric abnormalities are caused by the swelling of the astrocytes in the brain due to the accumulation of several factors, such as ammonia, serotonin, and shortchain fatty acids [2]. In particular, an increased blood ammonia concentration is a major factor for overt HE because of the inability of the systemic circulation to metabolize blood ammonia in patients with liver cirrhosis and/or a PSS [3]. Therefore, the current therapeutic approaches in HE rely on strategies to lower ammonia $[4,5]$.

The practice guideline for HE recommends lactulose as the first choice to treat episodic overt $\operatorname{HE}[5,6]$. For patients who have experienced one or more episodes of overt HE after administration of lactulose treatment, rifaximin is recommended as an add-on therapy for the prevention of overt HE recurrence [5]. Rifaximin is a nonabsorbable antibiotic for a broad spectrum that suppresses the absorption of ammonia by reducing the intestinal bacteria that produce ammonia [7]. Most studies have reported that rifaximin added to lactulose demonstrates improved QOL in patients with overt HE [8].

Thus, the current strategy for improving hyperammonemia is reducing ammoniagenesis and its absorption from the gut. However, the clinical usefulness of ammonia removal by upregulating ureagenesis through treatment with urea cycle supplementation has been reported $[8,9]$. In particular, L-carnitine supplementation activates the urea cycle and ammonia removal by upregulating ureagenesis [10-12]. Clinically, L-carnitine supplementation has been reported to reduce extraintestinal ammonia production and increase ammonia detoxification capacity in patients with cirrhosis [13].

The current therapeutic standard for overt HE to maintain remission is suppressing ammoniagenesis in the gastrointestinal tract using nonabsorbable disaccharides and a nonabsorbable antibiotic [14]. However, some patients with severe hepatic failure experience relapse of overt HE, irrespective of receiving a combination of rifaximin and lactulose. L-Carnitine is considered an additional agent in patients nonresponsive to the standard therapy for overt HE. Data regarding the additional effect on the recurrence of $\mathrm{HE}$ after oral L-carnitine administration are scarce. The present study aimed to assess the additional effects after administration of L-carnitine for patients who had overt $\mathrm{HE}$ and received the standard therapy.

\section{Patients and Methods}

\section{Eligibility Criteria}

Patients who met all of the following criteria were included: (1) grade I or II HE according to the West Haven Criteria, (2) one or more occurrences of overt $\mathrm{HE}$ after administration of nonabsorbable disaccharides, and ( 3$) \geq 20$ years of age. Liver cirrhosis and PSS were diagnosed based on the patients' imaging and laboratory tests. Patients' exclusion criteria were (1) acute hepatitis and exacerbation of chronic hepatitis, (2) poorly controlled hepatocellular carcinoma, (3) history of malignancies other than hepatocellular carcinoma, and (4) severe renal and/or heart failure.

\section{Study Design}

This open-label, randomized study was conducted at 2 centers from September 2016 through December 2020 and comprised a screening visit and a 12 -week treatment period. Patients who met the eligibility criteria for clinical trials were randomized in a 1:1 ratio to group A (additional rifaximin) or group B (additional Lcarnitine and rifaximin). Group A received 1,200 mg 3 times per day of rifaximin from the randomization. Group B received 1,500 $\mathrm{mg}$ of L-carnitine and 1,200 $\mathrm{mg}$ of rifaximin 3 times per day. The patients were followed up once every 4 weeks at our research facility after receiving these clinical drug therapies. At each follow-up, the patients' physical characteristics, medications, and blood test results were assessed. The final dosing day was defined as the day that the patient completed or discontinued the treatment. When the enrolled patients had severe adverse events (AEs) and/or HE exacerbation, the discontinuation and hospitalization decision depended on the clinical investigator. During the trial period, all participants received dietary education from dieticians who recommended moderate protein intake but did not recommend protein reduction.

\section{Evaluations}

This trial aimed to assess the additional effect of oral L-carnitine administration on overt $\mathrm{HE}$ in patients also receiving standard therapy. According to the phase III trial, the primary endpoints in both groups were the change in the portal systemic encephalopathy (PSE) index and blood ammonia levels after those baseline levels through the duration of the study. Secondary endpoints included the hospital admission rate and changes in laboratory data after administration of the trial drug. Table 1 shows the results of each item using the PSE index, which is calculated as PSE index $=(3 \times$ $\mathrm{HE}$ coma score + blood ammonia score + asterixis score + NCT-A score)/24.

Modified PSE index was measured because the original PSE index was using arterial blood samples to assess the blood ammonia concentrations. HE coma score, asterixis grade, and number of connection tests were assessed by the same clinical investigator.

The laboratory data included the hemoglobin level, platelet count, prothrombin time (PT), serum albumin, alanine aminotransferase (ALT), aspartate aminotransferase (AST), total bilirubin, serum creatinine, blood urea nitrogen (BUN), hemoglobin Alc (HbA1c), serum sodium, serum potassium levels, and the branched-chain amino acid tyrosine ratio (BTR). We also assessed the patients' AEs, which were graded using the Common Terminology Criteria for Adverse Events, version 4.0, and assessed after administering the trial drugs. 
Table 1. Scores and calculation criteria for the portal systemic encephalopathy index

\begin{tabular}{|c|c|c|c|c|}
\hline Score & Hepatic encephalopathy coma score & $\begin{array}{l}\text { Blood } \\
\text { ammonia } \\
\text { score, } \mu \mathrm{g} / \mathrm{dL}\end{array}$ & Asterixis score & $\begin{array}{l}\text { NCA-A } \\
\text { score, s }\end{array}$ \\
\hline 0 & No abnormality & $\leq 66$ & No asterixis observed & $<30$ \\
\hline 1 & $\begin{array}{l}\text { Inversion of sleep-wake rhythm } \\
\text { Euphoria and/or occasional depression } \\
\text { Negligent attitude with shortened attention span }\end{array}$ & $67-99$ & $\begin{array}{l}\text { No spontaneous asterixis observed, but it occurs } \\
\text { slightly when the subject is in a tremor-producing } \\
\text { posture }\end{array}$ & $30.1-50.0$ \\
\hline 2 & $\begin{array}{l}\text { Disorientation (time/place) and confusion (mixing things up) } \\
\text { Inappropriate behaviors (such as throwing away money or } \\
\text { discarding cosmetics in a trash can) } \\
\text { Occasional somnolent tendency (able to open eyes to normal } \\
\text { calls and converse) } \\
\text { Makes impolite remarks, but follows the doctors' instructions }\end{array}$ & $100-132$ & $\begin{array}{l}\text { No spontaneous asterixis observed, but it occurs } \\
\text { easily when the subject is in a tremor-producing } \\
\text { posture }\end{array}$ & $50.1-80.0$ \\
\hline 3 & $\begin{array}{l}\text { State of excitation and/or delirium, showing defiant behavior } \\
\text { Somnolent tendency (sleeping most of the time) } \\
\text { Able to open eyes in response to stimulation but does not or } \\
\text { cannot follow the doctors' instructions (respond to simple } \\
\text { orders) }\end{array}$ & $133-165$ & $\begin{array}{l}\text { Asterixis occurs without the subject in a tremor- } \\
\text { producing posture }\end{array}$ & $80.1-120$ \\
\hline 4 & $\begin{array}{l}\text { Coma (complete loss of consciousness) } \\
\text { Response to painful stimuli } \\
\text { Deep coma - no response to painful stimuli }\end{array}$ & $\geq 166$ & $\begin{array}{l}\text { Asterixis occurs almost continuously without the } \\
\text { subject in a tremor-producing posture or impossible } \\
\text { to perform }\end{array}$ & $>120$ \\
\hline
\end{tabular}

Fig. 1. Study flow diagram. HE, hepatic encephalopathy.

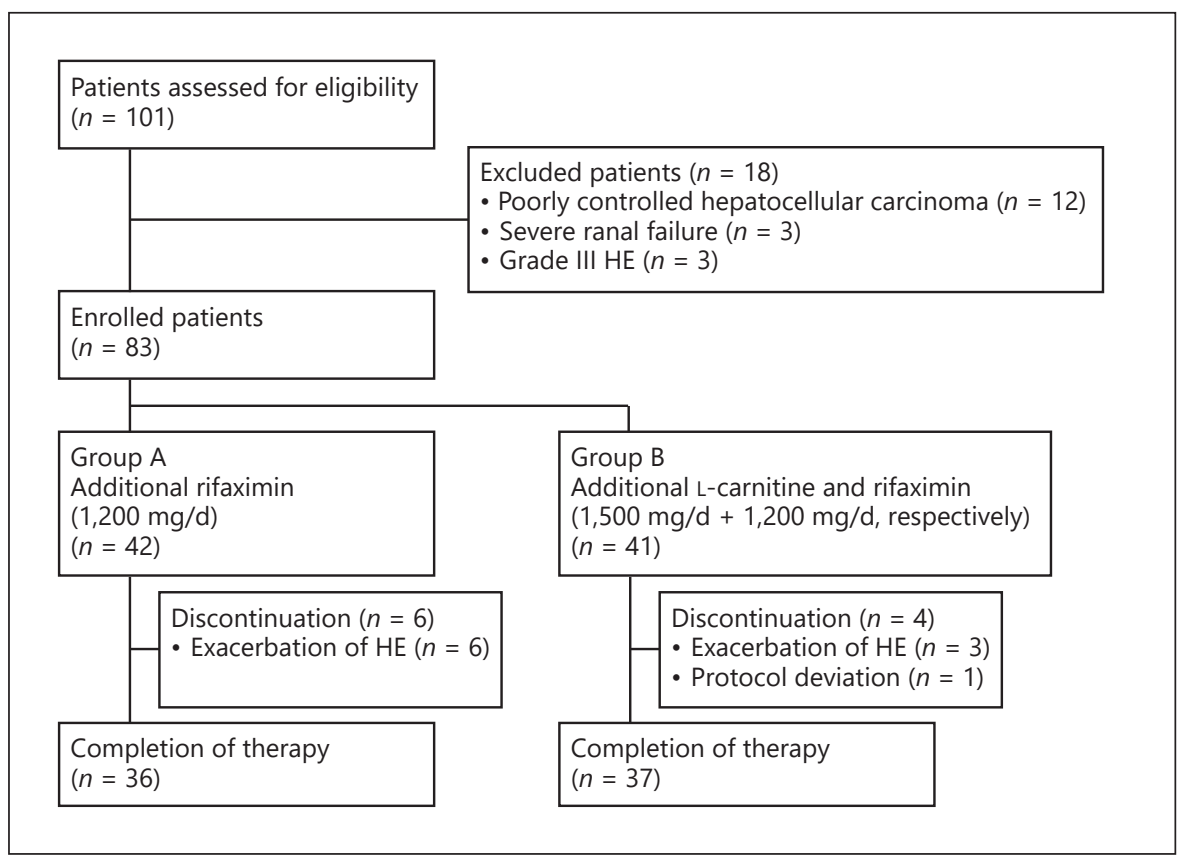

\section{Statistical Analyses}

The required sample size was estimated from previous studies [15]. Based on the clinical effects of L-carnitine on the blood ammonia concentration, the sample size for this trial to generate a $95 \%$ confidence level and a power of $80 \%$ was determined to be 46 patients per group, and the actual target sample size was set at 50 patients per group to allow for possible discontinuation. Interim analyses were planned when the clinical period of 4 years had lapsed, and the stopping rules were applied for the interim analyses based on $p$ values at primary endpoints. As a result, this trial was discontinued because of the statistical stopping rules of the interim analyses. 
Table 2. Patients' baseline characteristics

\begin{tabular}{llll}
\hline & $\begin{array}{l}\text { Group A }(n=42) \\
\text { additional rifaximin }\end{array}$ & $\begin{array}{l}\text { Group B }(n=41) \\
\text { additional L-carnitine } \\
\text { and rifaximin }\end{array}$ & $p$ value \\
\hline Age, years & & $68.0 \pm 10.5$ & 0.319 \\
Gender: male, $N$ (\%) & $65.6 \pm 11.6$ & $28(68.3)$ & 1.000 \\
Weight, kg & $29(69.0)$ & $66.7 \pm 10.7$ & 0.766 \\
BMI, kg/m & $65.9 \pm 13.7$ & $24.5 \pm 3.6$ & 0.775 \\
Etiology: virus/ALC/NASH/etc & $24.5 \pm 4.5$ & $7 / 13 / 12 / 9$ & 0.928 \\
History of liver cancer, $N(\%)$ & $8 / 13 / 14 / 7$ & $7(17.1)$ & 1.000 \\
Child-Pugh score & $8(19.0)$ & $8.67 \pm 1.6$ & 0.592 \\
Child-Pugh class: A/B/C, $N$ & $8.46 \pm 1.5$ & $1 / 28 / 12$ & 0.850 \\
PSE index & $1 / 26 / 15$ & $0.37 \pm 0.09$ & 0.740 \\
Blood ammonia concentration, $\mu$ g/dL & $0.35 \pm 0.09$ & $130.6 \pm 39.1$ & 0.383 \\
Mental state of HE: grade I/II, $N(\%)$ & $126.6 \pm 49.0$ & $28 / 13$ & 0.817 \\
Asterixis grade, $0 / 1 / 2 / 3$ & $27 / 15$ & $33 / 8 / 0 / 0$ & 1.000 \\
NCT-A, s & $33 / 9 / 0 / 0$ & $59.1 \pm 13.0$ & 0.470 \\
History of admission from HE, $N$ & $58.9 \pm 12.5$ & $31 / 11$ & 0.885 \\
Portal systemic shunt, $N$ (\%) & $33 / 10$ & $27(64.2)$ & 0.620 \\
Concomitant medication & $29(67.4)$ & & 0.651 \\
$\quad$ Nonabsorbable disaccharides, $N(\%)$ & $39(92.9)$ & $38(92.7)$ & 0.810 \\
$\quad$ Branched-chain amino acid, $N(\%)$ & $29(69.0)$ & $30(73.2)$ & 0.474 \\
Prothrombin time, $\%$ & $66.0 \pm 20.2$ & $63.2 \pm 19.2$ & 0.210 \\
Serum albumin, g/dL & $3.10 \pm 0.61$ & $3.3 \pm 0.54$ & 0.393 \\
Serum creatinine, mg/dL & $0.89 \pm 0.24$ & $0.85 \pm 0.26$ & 0.937 \\
Aspartate aminotransferase, IU/L & $49.5 \pm 25.4$ & $50.2 \pm 37.8$ & 0.675 \\
Alanine aminotransferase, IU/L & $32.4 \pm 15.5$ & $34.2 \pm 19.3$ & 0.957 \\
Total bilirubin, g/dL & $1.9 \pm 1.0$ & $1.9 \pm 1.0$ & \\
\hline
\end{tabular}

ALC, alcohol; NST, number connection test; NASH, nonalcoholic steatohepatitis; HE, hepatic encephalopathy.

The analyses were conducted for the full analysis set, which was defined as all of the patients receiving the trial drugs at least once. Differences of $p$ values $<0.05$ were considered significant. The data were expressed as means and standard deviations. Missing data on the final evaluation day were input based on the most recent data obtained after the study began. Categorical variables were compared using $\chi^{2}$ tests, continuous variables were compared using the unpaired $t$ test, and paired data were compared using $t$ tests. Blood ammonia concentrations at the final evaluation were assessed using Tukey's honestly significant difference test with baseline values as the covariate. Statistical analyses were performed using IBM $^{\circledR}$ SPSS $^{\circledR}$ software, version 24.0 (IBM Corporation, Armonk, NY, USA). The statistical analyses were reviewed by Statista Corporation, Kyoto, Japan.

\section{Results}

\section{Patients' Characteristics}

A total of 101 patients were assessed for clinical trials. Of these, 18 patients did not meet the above inclusion criteria: 12 patients had poorly controlled hepatocellular carcinoma, 3 patients had severe renal failure, and 3 pa- tients had grade III HE. The remaining 83 patients were randomized to either group A $(n=42)$ or group B $(n=$ 41) (Fig. 1). The clinical trial was discontinued in 10 patients $(12.0 \%)$ because of HE exacerbation $(n=9)$ and protocol deviations $(n=1)$.

Table 2 summarizes the patients' baseline characteristics. Differences between the 2 groups were not significant with respect to age, weight, presence of hepatocellular carcinoma, etiology of liver cirrhosis, rate of hepatic ascites, Child-Pugh score, hemoglobin level, platelet count, PT, BTR, serum albumin, serum creatinine, AST, ALT, or total bilirubin.

\section{Changes in the Blood Ammonia Levels}

In group $A$, the changes in the blood ammonia levels at weeks 4,8 , and 12 from baseline were $-11.5 \pm 13.6$, $-21.2 \pm 24.7$, and $-30.9 \pm 25.1 \mu \mathrm{g} / \mathrm{dL}(p=0.379, p=0.003$, and $p=0.016$, respectively). In group $B$, the changes in the blood ammonia levels at weeks 4,8 , and 12 from baseline were $-29.6 \pm 26.1,-29.8 \pm 25.9$, and $-31.2 \pm 24.6 \mu \mathrm{g} /$ $\mathrm{dL}$, respectively (all $p<0.001$ ). After administering the 
Fig. 2. Blood ammonia levels $(\mu \mathrm{g} / \mathrm{dL})$. Data are expressed as mean \pm standard deviation. Comparisons between the final values and the baseline values for both groups were performed using the nonpaired $t$ test. Comparisons between the groups were performed using ANOVA.
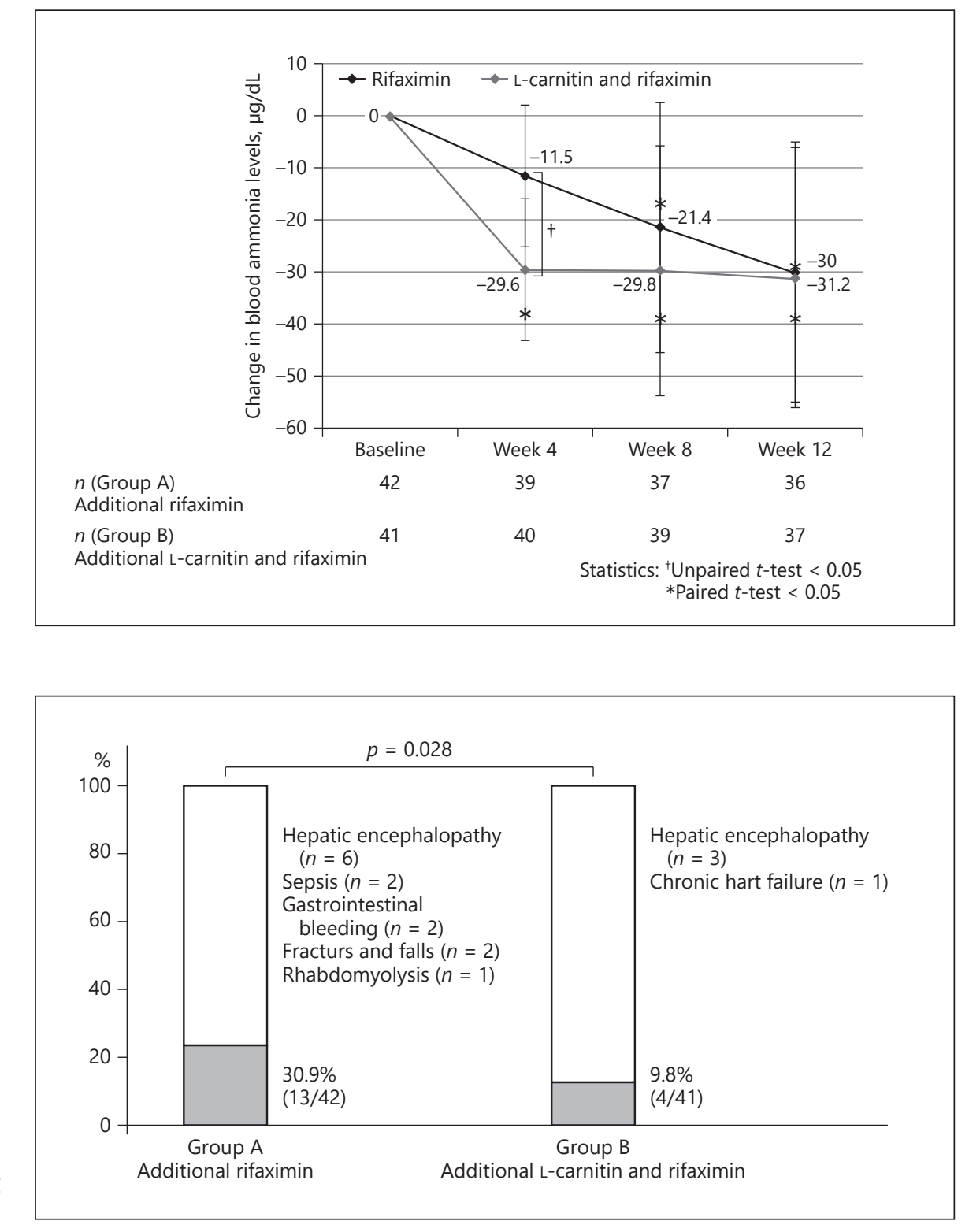

Fig. 3. Influence for the admission rates. Categorical variables were compared using $\chi^{2}$ tests. trial drug at 4 weeks, a significant reduction in the blood ammonia levels was observed in the 2 groups $(p=0.009)$. However, differences between the groups regarding changes in the blood ammonia levels on the final dosing day were not significant ( $p=0.173$ ) (Fig. 2).

\section{Changes in the PSE Index}

In group A, the PSE index decreased from $0.35 \pm 0.09$ at baseline to $0.27 \pm 0.11$ on the final evaluation day. The changes in PSE on the final evaluation day from baseline were significantly decreased $(p<0.001)$. In group $B$, the PSE index decreased from $0.37 \pm 0.09$ at baseline to 0.24 \pm 0.11 on the final evaluation day. The changes in the PSE index on the final evaluation day from baseline were significantly decreased $(p<0.001)$. After administering the trial drug, a trend was seen in the greater reduction of the PSE index in group B than that in group A. However, there was no significant reduction in the PSE index in group A compared to that in group B $(p=0.202)$.

\section{Influence on the Hospital Admission Rates during the Treatment Span}

During the treatment period, the hospital admission rates in groups A and B were $30.9 \%(13 / 42)$ and $9.8 \%$ (4/41), respectively. Additional L-carnitine significantly reduced the admission rate $(p=0.028)$ (Fig. 3$)$. In group 
Table 3. Change in laboratory data after administration of the trial drug

\begin{tabular}{|c|c|c|c|c|c|c|}
\hline & \multicolumn{3}{|c|}{ Group A: additional rifaximin } & \multicolumn{3}{|c|}{ Group B: additional L-carnitine and rifaximin } \\
\hline Hemoglobin, g/dL & $12.3 \pm 1.92$ & $12.1 \pm 2.18$ & 0.823 & $12.1 \pm 2.11$ & $11.9 \pm 2.4$ & 0.243 \\
\hline Platelets, $\times 10^{3} / \mu \mathrm{L}$ & $11.1 \pm 5.66$ & $9.92 \pm 3.89$ & 0.180 & $11.0 \pm 6.48$ & $12.8 \pm 13.1$ & 0.284 \\
\hline PT time, $\%$ & $66.0 \pm 20.2$ & $67.1 \pm 19.4$ & 0.526 & $63.2 \pm 19.2$ & $65.9 \pm 17.1$ & 0.382 \\
\hline Albumin, $\mathrm{g} / \mathrm{dL}$ & $3.10 \pm 0.61$ & $3.13 \pm 0.64$ & 0.784 & $3.29 \pm 0.54$ & $3.30 \pm 0.66$ & 0.851 \\
\hline AST, IU/L & $49.5 \pm 25.4$ & $53.1 \pm 37.8$ & 0.859 & $50.2 \pm 37.8$ & $47.6 \pm 24.4$ & 0.580 \\
\hline $\mathrm{ALT}, \mathrm{IU} / \mathrm{L}$ & $32.4 \pm 15.5$ & $31.6 \pm 19.2$ & 0.899 & $34.2 \pm 19.3$ & $29.9 \pm 14.1$ & 0.436 \\
\hline T-Bil, g/dL & $1.87 \pm 0.97$ & $2.04 \pm 1.17$ & 0.207 & $1.91 \pm 1.06$ & $1.95 \pm 1.21$ & 0.875 \\
\hline HBA1c, \% & $5.92 \pm 1.45$ & $6.01 \pm 1.89$ & 0.810 & $5.70 \pm 1.13$ & $5.57 \pm 1.21$ & 0.703 \\
\hline BTR & $3.47 \pm 1.68$ & $3.52 \pm 2.01$ & 0.706 & $3.95 \pm 2.80$ & $4.42 \pm 1.63$ & 0.331 \\
\hline
\end{tabular}

PT, prothrombin time; BUN, blood urea nitrogen; ALT, alanine aminotransferase; AST, aspartate aminotransferase; T-Bil, total bilirubin; HbA1c, hemoglobin A1c; BTR, branched-chain amino acid tyrosine ratio.

Table 4. Adverse events

\begin{tabular}{|c|c|c|c|c|}
\hline & \multicolumn{2}{|c|}{$\begin{array}{l}\text { Group A: additional } \\
\text { rifaximin }\end{array}$} & \multicolumn{2}{|c|}{$\begin{array}{l}\text { Group B: additional } \\
\text { L-carnitine and rifaximin }\end{array}$} \\
\hline & any grade & $\begin{array}{l}\text { grades } \\
3 \text { or } 4\end{array}$ & any grade & $\begin{array}{l}\text { grades } \\
3 \text { or } 4\end{array}$ \\
\hline Total & $5(11.9)$ & $1(2.3)$ & $9(21.9)$ & 0 \\
\hline Rhabdomyolysis & $1(2.3)$ & $1(2.3)$ & 0 & 0 \\
\hline Nausea & $1(2.3)$ & 0 & $2(4.8)$ & 0 \\
\hline Itching & $1(2.3)$ & 0 & $2(4.8)$ & 0 \\
\hline Diarrhea & $1(2.3)$ & 0 & $1(2.4)$ & 0 \\
\hline Headache & 0 & 0 & $1(2.4)$ & 0 \\
\hline Taste disorder & $1(2.3)$ & 0 & 0 & 0 \\
\hline Heart failure & 0 & 0 & $1(2.4)$ & 0 \\
\hline Abdominal pain & 0 & 0 & $1(2.4)$ & 0 \\
\hline Jaundice & 0 & 0 & $1(2.4)$ & 0 \\
\hline
\end{tabular}

A, 6 patients had HE-related admissions, 2 patients had sepsis, 2 patients sustained trauma after falling down the stairs, and 2 patients had gastrointestinal bleeding. Another patient was admitted for rhabdomyolysis. Patients with HE-related admissions exhibited consciousness disorder in weeks 4 and 8 ( $n=2$ and 5 , respectively).

In group B, 3 patients had HE-related admissions. One patient was admitted because of sustaining a subdural hematoma after falling on stairs. Another patient had chronic heart failure. In one other patient with HE-related admission, consciousness disorder manifested within week $8(n=1)$.

\section{Pharmacological Effects}

In group $\mathrm{A}$, differences at baseline and on the final evaluation day $(3.1 \pm 0.6$ vs. $3.1 \pm 0.6 \mathrm{~g} / \mathrm{dL}, p=0.784)$ were not significant (Table 3). In group B, differences at baseline and on the final evaluation day $(3.3 \pm 0.5$ vs. $3.3 \pm 0.7$ $\mathrm{g} / \mathrm{dL}, p=0.851)$ were not significant. There were no differences, in either group, between the baseline and the final dosing day in the hemoglobin level, platelet count, PT, BUN, albumin, BTR, serum creatinine, ALT, total bilirubin, or HbAlc.

\section{Safety}

Treatment-related AEs during the trial span were observed in 5 patients $(11.9 \%)$ in group $\mathrm{A}$ and 9 patients $(21.9 \%)$ in group B (Table 4$)$. Differences in the frequency of AEs between the groups were not significant ( $p=$ 0.254 ). There were no deaths directly attributable to the trial drug; however, 1 patient experienced a severe $\mathrm{AE}$ (rhabdomyolysis), leading to hospital admission. 
In the L-carnitine administration therapy group, the AEs were nausea, itching, diarrhea, headache, heart failure, and abdominal pain. In the rifaximin-only group, AEs were rhabdomyolysis, nausea, itching, diarrhea, and taste disorder. Nausea and itching were more common in the L-carnitine group than in the rifaximin-only group.

\section{Discussion}

To our knowledge, this is the first report of the additional effects of L-carnitine for patients with overt $\mathrm{HE}$ who received the standard therapy. L-Carnitine has an essential role in energy metabolism in the transport of longchain fatty acids across the inner mitochondrial membrane [15-17]. Progression of fibrosis leads to carnitine deficiency because the liver is the major organ for carnitine metabolism and distribution [18]. Low blood levels of acetyl-L-carnitine in liver cirrhosis reduce the activities of the key enzymes of the urea cycle, which converts toxic ammonia into urea for excretion through urine. L-Carnitine supplementation activates the urea cycle and the activation of ammonia removal by upregulation of ureagenesis $[19,20]$.

Therefore, additional L-carnitine can reduce the blood ammonia levels in patients with HE. As a result, a significantly greater reduction in the blood ammonia levels at 4 weeks was observed in the additional L-carnitine group compared with only additional rifaximin. Previously, Hanai et al. [21] reported that the most change in ammonia concentrations was at 4 weeks after L-carnitine supplementation in patients with liver cirrhosis. This result was corroborated in the present study. We suppose that ammonia removal by upregulating ureagenesis by treatment with supplemental drugs affecting the urea cycle is a quick response compared with the reduction of ammoniagenesis after administering rifaximin.

However, the difference in the blood ammonia levels on the final dosing days in the 2 groups was not significant. Some researchers have reported that ammonia levels do not influence therapy in clinical practice [22]. Therefore, we also evaluated the PSE index, which is critical for anticipating and recognizing complications [8]. Nevertheless, differences between the 2 groups were not significant with respect to the PSE index, although a trend was seen in the greater reduction of the PSE index in the additional L-carnitine group than that in the standard therapy group. This result was unexpected; however, ammonia removal by upregulating ureagenesis through treatment with L-carnitine supplementation is not insig-

Combination Therapy with Rifaximin and L-Carnitine nificant, that is, it does offer some substantial benefit to the patient.

Clinically, an important goal in treating patients with cirrhosis is preventing hospitalization-related complications, including $\mathrm{HE}$, hepatic ascites, gastrointestinal bleeding, and sarcopenia. This clinical study has demonstrated that the additional L-carnitine therapy, compared with a standard rifaximin therapy, significantly reduced hospitalization risk in patients with overt HE. We suppose that the early improvement of ammonia levels can help reduce the frequency of hospitalization. Especially, early reduction of the blood ammonia level is important for patients with uncontrollable overt HE unless there is administration of nonabsorbable disaccharides. One patient with overt HE may show a rapid response favorably to additional rifaximin, whereas another patient with $\mathrm{HE}$ of comparable severity may require the addition of L-carnitine and rifaximin to achieve early reduction of the blood ammonia level. In fact, there was a small number of hospitalizations in group $\mathrm{B}$, which revealed the improvement of blood ammonia levels within 8 weeks, as shown in Figure 2. Symptoms of overt HE decrease activity, leading to improper nutrition, immunodeficiency, and sarcopenia, leading to more frequent hospitalizations [9]. Furthermore, L-carnitine supplementation has been reported to improve sarcopenia. Hyperammonemia leads to upregulate the expression of myostatin, which suppresses the synthesis of skeletal muscle [23]. Therefore, we suggest an approach to therapy aimed at rapidly achieving early reduction of the blood ammonia level. The faster the improvement in blood ammonia levels, the greater the benefits to help reduce the frequency of hospitalization.

We also expected that L-carnitine will improve serum albumin levels after administration during the span of the trial. That result was because a systematic review and meta-analysis for liver cirrhosis patients receiving L-carnitine reported the improvement of the various abnormal levels related to the liver function and renal function, including albumin, BUN, AST, ALT, total bilirubin, and PT [11]. However, this present clinical study showed no effects on albumin, total bilirubin, and PT in patients with HE. This controversy may be due to the short follow-up for the enrolled patients.

According to the safety profile during this clinical study, the incidences of any and particularly severe AEs were statistically similar in both these groups. This study showed that L-carnitine supplementation has a low risk of interactions with rifaximin. L-Carnitine tends to have mild side effects, including nausea, headache, diarrhea, 
and restlessness. Typical side effects of L-carnitine therapy are rapid heart rate and increased blood pressure [11]. In this study, heart failure was seen as an $\mathrm{AE}$ in the additional L-carnitine group. The use of $\mathrm{L}$-carnitine is restricted if the patient experiences cardiac dysfunction.

Our final goal was to create an appropriate strategy for patients with severe hepatic failure relapse with overt HE, irrespective of whether rifaximin was added to lactulose. Combination therapy to reduce ammoniagenesis and upregulate ureagenesis can benefit patients with overt HE [24]. However, the effectiveness of combination therapy has not yet been systematically investigated and remains poorly understood. The effects of the combination treatment with different mechanisms warrant further investigation.

The present study has several limitations. First, clinically meaningful results and reducing the risk of hospitalization were secondary end points. A re-examination of this is required. Second, the appropriate doses of L-carnitine may differ among patients. The enrolled patients had relatively severely impaired liver function. If they had presented with milder liver dysfunction, the dose of the L-carnitine would likely have been significantly different. Third, this clinical trial was an open-label study. Therefore, there was an intentional bias regarding discontinuing treatment or continuing hospitalization for these patients. Finally, this study was not a multicenter study. This might have led to a selection bias.

\section{Conclusion}

The current study demonstrated that additional L-carnitine had a beneficial effect on the hospital admission rate in patients who received rifaximin and lactulose. LCarnitine with standard therapy could be a useful treatment for patients with overt HE.

\section{Acknowledgment}

We thank Robert E. Brandt, Founder, CEO, and CME, of MedEd Japan, for editing and formatting the manuscript.

\section{Statement of Ethics}

The research protocol for this study follows the provisions of the Declaration of Helsinki and was approved by the Institutional Review Board Ethics Committee at all participating institutes and registeredin the UMINClinical Trials Registryas UMIN000027511. All patients were informed regarding the relevance of the analysis and provided written informed consent.

\section{Conflict of Interest Statement}

The authors have no conflicts of interest to declare.

\section{Funding Sources}

The authors did not receive any funding.

\section{Author Contributions}

Kubota K., Uojima H., Shao X., Iwasaki S., Hidaka H., Wada N., Nakazawa T., Shibuya A., Kako M., and Koizumi W. contributed equally to this work; Uojima H. and Kubota K. collected and analyzed the data; Uojima $\mathrm{H}$. drafted the manuscript; Hidaka $\mathrm{H}$. designed and supervised the study; Shao X., Iwasaki S., Wada N., Kubota K., Nakazawa T., Shibuya A., Kako M., and Koizumi W. offered technical or material support. All authors discussed the results and approved the final manuscript for publication.

\section{Data Availability Statement}

The data that support the findings of this study are available from the corresponding author upon reasonable request.

\section{References}

1 Wijdicks EF. Hepatic encephalopathy. N Engl J Med. 2016;375(17):1660-70.

2 Bajaj JS. Hepatic encephalopathy: classification and treatment. J Hepatol. 2018;68(4): $838-9$.

3 Vilstrup H, Amodio P, Bajaj J, Cordoba J, Ferenci $\mathrm{P}$, Mullen KD, et al. Hepatic encephalopathy in chronic liver disease: 2014 Practice Guideline by the American Association for the Study of Liver Diseases and the European Association for the Study of the Liver. Hepatology. 2014;60(2):715-35.
4 Suraweera D, Sundaram V, Saab S. Evaluation and management of hepatic encephalopathy: current status and future directions. Gut Liver. 2016;10(4):509-19.

5 Patidar KR, Bajaj JS. Covert and overt hepatic encephalopathy: diagnosis and management. Clin Gastroenterol Hepatol. 2015;13(12): 2048-61.

6 Hudson M, Schuchmann M. Long-term management of hepatic encephalopathy with lactulose and/or rifaximin: a review of the evidence. Eur J Gastroenterol Hepatol. 2019;31(4):434-50.
7 Flamm SL. Rifaximin treatment for reduction of risk of overt hepatic encephalopathy recurrence. Therap Adv Gastroenterol. 2011;4(3): 199-206.

8 Suzuki K, Endo R, Takikawa Y, Moriyasu F, Aoyagi Y, Moriwaki $\mathrm{H}$, et al. Efficacy and safety of rifaximin in Japanese patients with hepatic encephalopathy: a phase II/III, multicenter, randomized, evaluator-blinded, active-controlled trial and a phase III, multicenter, open trial. Hepatol Res. 2018; 48(6):411-23. 
9 Tani J, Morishita A, Sakamoto T, Takuma K, Nakahara M, Fujita K, et al. L-carnitine reduces hospital admissions in patients with hepatic encephalopathy. Eur J Gastroenterol Hepatol. 2021;32(2):288-93.

10 Ferenci P, Lockwood A, Mullen K, Tarter R, Weissenborn K, Blei AT. Hepatic encephalopathy: definition, nomenclature, diagnosis, and quantification: final report of the working party at the 11th World Congresses of Gastroenterology, Vienna, 1998. Hepatology. 2002; 35(3):716-21.

11 Abbasnezhad A, Choghakhori R, Kashkooli S, Alipour M, Asbaghi O, Mohammadi R. Effect of L-carnitine on liver enzymes and biochemical factors in hepatic encephalopathy: a systematic review and meta-analysis. J Gastroenterol Hepatol. 2019;34(12):2062-70.

12 Bass NM, Mullen KD, Sanyal A, Poordad F, Neff G, Leevy CB, et al. Rifaximin treatment in hepatic encephalopathy. $\mathrm{N}$ Engl J Med. 2010;362(12):1071-81.

13 Wang Z, Chu P, Wang W. Combination of rifaximin and lactulose improves clinical efficacy and mortality in patients with hepatic encephalopathy. Drug Des Devel Ther. 2019; 13:1-11.
14 Sanchez-Delgado J, Miquel M. [Role of rifaximin in the treatment of hepatic encephalopathy]. Gastroenterol Hepatol. 2016;39(4):28292.

15 Ohara M, Ogawa K, Suda G, Kimura M, Maehara $\mathrm{O}$, Shimazaki T, et al. L-carnitine suppresses loss of skeletal muscle mass in patients with liver cirrhosis. Hepatol Commun. 2018; 2(8):906-18.

16 Hiraoka A, Kiguchi D, Ninomiya T, Hirooka M, Abe M, Matsuura B, et al. Can L-carnitine supplementation and exercise improve muscle complications in patients with liver cirrhosis who receive branched-chain amino acid supplementation? Eur J Gastroenterol Hepatol. 2019;31(7):878-84.

17 Sato S, Namisaki T, Furukawa M, Saikawa S, Kawaratani H, Kaji K, et al. Effect of L-carnitine on health-related quality of life in patients with liver cirrhosis. Biomed Rep. 2020;13(6): 65.

18 Martí-Carvajal AJ, Gluud C, Arevalo-Rodriguez I, Martí-Amarista CE. Acetyl-L-carnitine for patients with hepatic encephalopathy. Cochrane Database Syst Rev. 2019;1(1): CD011451.
19 Ebadi M, Bhanji RA, Mazurak VC, MontanoLoza AJ. Sarcopenia in cirrhosis: from pathogenesis to interventions. J Gastroenterol. 2019;54(10):845-59.

20 Nakanishi H, Kurosaki M, Tsuchiya K, Nakakuki N, Takada H, Matsuda S, et al. L-carnitine reduces muscle cramps in patients with cirrhosis. Clin Gastroenterol Hepatol. 2015; 13(8):1540-3.

21 Hanai T, Shiraki M, Imai K, Suetugu A, Takai $\mathrm{K}$, Shimizu M. Usefulness of carnitine supplementation for the complications of liver cirrhosis. Nutrients. 2020;12(7):1915.

22 Haj M, Rockey DC. Ammonia levels do not guide clinical management of patients with hepatic encephalopathy caused by cirrhosis. Am J Gastroenterol. 2020;115(5):723-8.

23 Miyaaki H, Kobayashi H, Miuma S, Fukusima M, Sasaki R, Haraguchi M, et al. Blood carnitine profiling on tandem mass spectrometry in liver cirrhotic patients. BMC Gastroenterol. 2020;20(1):41.

24 Flamm SL. Complications of cirrhosis in primary care: recognition and management of hepatic encephalopathy. Am J Med Sci. 2018; 356(3):296-303. 